\title{
Pioglitazone attenuates endotoxin-induced acute lung injury by reducing neutrophil recruitment
}

\author{
Jochen Grommes*,\#, Mathias Mörgelin ${ }^{\star}$ and Oliver Soehnlein*,+,
}

ABSTRACT: Treatment of acute lung injury (ALI) remains an unsolved problem in intensive care medicine. Activation and recruitment of neutrophils are regarded as key mechanisms in the progression of ALI. As pioglitazone holds potent pleiotropic anti-inflammatory effects, we explored its effects during $A L I$.

C57BI/6 mice were exposed to aerosolised lipopolysaccharides (LPSs) $\left(500 \mu \mathrm{g} \cdot \mathrm{mL}^{-1}\right)$ and their alveolar, interstitial and intravascular neutrophils were assessed $4 \mathrm{~h}$ later. Lung permeability changes were evaluated by fluorescein isothiocyanate-dextran clearance and protein content in the bronchoalveolar lavage fluid. In vitro, human isolated neutrophils were pretreated with piolitazone $(10 \mu \mathrm{M}$, for 1 or $3 \mathrm{~h})$ and then activated with $N$-formyl-L-methionyl-L-leucyl-Lphenylalanine. Neutrophil activation, adhesion, release and formation of reactive oxygen species (ROS) and phagocytosis were measured thereafter.

Pioglitazone treatment before or after induction of ALI significantly diminished alveolar (reduction by $73 \%$ and $67 \%$, respectively) and interstitial neutrophil influx (reduction by $55 \%$ and $63 \%$, respectively) and reduced lung permeability changes (reduction by $64 \%$ and $58 \%$, respectively) indicating a protective role of pioglitazone treatment in ALI. Moreover, pioglitazone significantly reduced degranulation and adhesion of neutrophils without affecting ROS formation and release or bacterial phagocytosis.

Pioglitazone reduces recruitment and activation of neutrophils thereby preventing LPS-induced ALI. Our results imply a potential role for pioglitazone treatment in the management of ALI.

KEYWORDS: Acute lung injury, neutrophils, pioglitazone

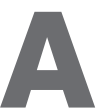

cute lung injury (ALI) is a life-threatening disease with an age-adjusted incidence of 86.2 per 100,000 persons a year [1] Despite all innovations in intensive care medicine, the mortality of ALI remains up to $40 \%$. ALI is characterised by an increased permeability of the alveolar-capillary barrier resulting in lung oedema with protein-rich fluid consequently leading to impairment of arterial oxygenation. Sepsis is a major cause for the development of ALI, wherein Gram-negative bacteria are predominant. Lipopolysaccharides (LPS) inhalation mimics human Gram-negative ALI, inducing neutrophil recruitment, pulmonary oedema and finally impairment of gas exchange [2]. Recruitment of neutrophils is a key event in the development of ALI [1, 3], leading to plasma leakage and deterioration of oxygenation. The importance of neutrophils in ALI is supported by studies where lung injury is abolished by the depletion of neutrophils [4, 5]. Much of the neutrophil-dependent ALI is mediated by granule proteins released from activated neutrophils. For example, azurocidin and $\alpha$-defensins were implied to directly alter changes in permeability $[6,7]$, whereas proteases of neutrophilic origin, such as neutrophil elastase, have been suggested to be important in the degradation of surfactant proteins, epithelial cell apoptosis, and coagulation [8, 9]. Moreover, neutrophils produce vast quantities of reactive oxygen species (ROS) and reactive nitrogen species. Besides their important antimicrobial effector function, neutrophil-derived oxidants promote deleterious pro-inflammatory effects, and thus are a major cause of neutrophil-dependent tissue injury in ALI [3].

Peroxisome proliferator-activated receptors (PPAR) are known as transcription factors that belong to the
AFFILIATIONS

*Institute for Molecular

Cardiovascular Research (IMCAR), RWTH Aachen,

\#Dept of Vascular Surgery, RWTH

Aachen, Aachen, and

+Institute for Cardiovascular

Prevention, Ludwig-Maximilians-

University, Munich, Germany.

-Division of Infection Medicine, Dept

of Clinical Sciences, Lund University,

Lund, Sweden.

${ }^{\S}$ Cardiovascular Research Institute Maastricht, Maastricht University,

Maastricht, The Netherlands.

CORRESPONDENCE

J. Grommes

Dept of Vascular Surgery

RWTH Aachen

Pauwelsstr. 30

52074 Aachen

Germany

E-mail: jgrommes@ukaachen.de

Received:

May 292011

Accepted after revision:

Nov 242011

First published online:

Jan 202012 
nuclear hormone receptor superfamily. PPARs are ligandactivated transcription factors, containing three isoforms $(\alpha, \beta$, and $\gamma$ ) being encoded by unique genes. Besides their importance in the regulation of both lipid and carbohydrate metabolism, PPARs, especially PPAR- $\alpha$ and PPAR- $\gamma$, have received much attention for their potent anti-inflammatory effects [10]. Previous studies have suggested that PPAR- $\gamma$ ligands reduce the expression of inflammatory cytokine genes and the production of inflammatory cytokines [11, 12]. Consequently, PPAR- $\alpha$ and PPAR- $\gamma$ agonists may be helpful in the treatment of acute inflammatory diseases, such as ALI [13]. In this context, several studies have proven a beneficial role for PPAR- $\gamma$ agonists in models of allergic airway inflammation and bleomycin-induced ALI [14, 15].

Although previous in vitro and in vivo studies have revealed the anti-inflammatory effects of pioglitazone, there is less known about the effects of pioglitazone on neutrophils in ALI. Recruitment of neutrophils, release of granule proteins and generation of ROS by neutrophils display key events in ALI and may be a suitable potential target for therapy. Thus, we address the effect of pioglitazone treatment in a model of neutrophil-dependent ALI.

\section{METHODS}

\section{Animals}

8-week-old male C57Bl/6 mice were obtained from Janvier SAS (Le Genest Saint Isle, France). Neutrophils were depleted by intraperitoneal injection of Ly6G-specific monoclonal antibody $1 \mathrm{~A} 8(100 \mu \mathrm{g}$ per mouse $12 \mathrm{~h}$ and $0 \mathrm{~h}$ before LPS inhalation; BioXcell, West Lebanon, NH, USA). Mice with intact white blood cell counts were treated with either pioglitazone ( $2 \mu \mathrm{g}$ per $\mathrm{g}$ of bodyweight) or $\mathrm{NaCl} 0.9 \%$ by intraperitoneal injection $12 \mathrm{~h}$ and $0 \mathrm{~h}$ before LPS inhalation ort $1 \mathrm{~h}$ after LPS inhalation, respectively. All experiments were approved by the local ethical authorities.

\section{Murine model of ALI}

Aerosolised LPS from Salmonella enteritidis (Sigma-Aldrich Co., St. Louis, MO, USA) dissolved in $0.9 \%$ saline $\left(500 \mu \mathrm{g} \cdot \mathrm{mL}^{-1}\right)$ was utilised to induce neutrophil infiltration in the lung. Six mice were exposed simultaneously to aerosolised LPS in a custom-built box $(22 \mathrm{~cm}$ in length $10 \mathrm{~cm}$ in diameter) connected to an air nebuliser (MicroAir; Omron Healthcare, Vernon Hills, IL, USA) for $30 \mathrm{~min}$. Eight control mice were exposed to saline aerosol. Neutrophil counts in the bronchoalveolar lavage fluid (BALF) and lung tissue (interstitium and pulmonary vasculature) were assessed $4 \mathrm{~h}$ after inhalation. $30 \mathrm{~min}$ before euthanasia, $5 \mu \mathrm{L}$ of anti-mouse-Ly-6G (Gr-1) fluorescein isothiocyanate (FITC) (Gr1; eBioscience, San Diego, CA, USA) and $100 \mu \mathrm{l}$ FITC-dextran $\left(30 \mathrm{mg} \cdot \mathrm{mL}^{-1}\right.$ FITC-dextran, $70 \mathrm{kDa}$; Sigma-Aldrich Co.) were applied via a tail-vein injection to label intravascular neutrophils. The mice were anesthetised with an intraperitoneal injection of ketamine (125 mg per $\mathrm{kg}$ body weight; Sanofi-Cefa GmbH, Düsseldorf, Germany) and xylazine (12.5 mg per kg body weight; Phoenix Scientific, St Joseph, MO, USA). The trachea was dissected and cannulated (PortexFineBore polythene tubing, $0.28 \mathrm{~mm}$ inner diameter/ $0.61 \mathrm{~mm}$ outer diameter; Smiths Medical International, Keene, $\mathrm{NH}$, USA). $5 \times 0.5 \mathrm{~mL}$ PBS was injected and withdrawn. Thereafter, the ribcage was opened by a midline incision and the pulmonary vasculature was rinsed with $15 \mathrm{~mL}$ ice-cold PBS with $0.5 \mathrm{mM}$ EDTA after cutting the inferior cava vein to facilitate exsanguination. The lungs were removed, minced and digested with liberase (1:20, $25 \mathrm{mg}$ Liberase $\mathrm{RI} \cdot \mathrm{mL}^{-1}$ aqua; Roche, Mannheim, Germany). Digested lungs were passed through a cell strainer $(70 \mu \mathrm{m}$; Miltenyi Biotec $\mathrm{GmbH}$, Bergisch Gladbach, Germany) and the resulting single-cell suspension was centrifuged for $5 \mathrm{~min}$ at $300 \mathrm{~g}$. The pellets were resuspended in $1 \mathrm{~mL}$ Hank's balanced salt solution with $0.3 \mathrm{mmol} \cdot \mathrm{L}^{-1}$ EDTA and $0.1 \%$ bovine serum albumin (BSA). BALF was centrifuged for $5 \mathrm{~min}$ at $300 \mathrm{~g}$ (fig. S1).

\section{Flow cytometry}

Cell pellets were labelled with PerCP-Cy5.5 anti-mouse Ly-6G, PE anti-mouse CD115, APC-Cy7 anti-mouse CD45 and APC anti-mouse F4/80 (eBioscience). Neutrophils were identified by their typical appearance in the forward scatter side scatter and as CD45+, CD115- and PerCP-Gr1+ cells (fig. S2). Within the lung, FITC-Gr1 antibody was used to distinguish between interstitial neutrophils (CD45+, CD115-, PerCP-Gr+, FITC-Gr1-) and intravascular neutrophils (CD45+, CD115-, PerCP-Gr1+, FITC-Gr1+). All flow cytometry studies were performed using a BD FACS Canto II (Becton Dickinson, San Jose, CA, USA) and data were analysed using FlowJo software (Tree Star, Ashland, OR, USA).

\section{Lung permeability}

FITC-dextran was used to assess vascular leakage. $100 \mu$ L FITCdextran $\left(30 \mathrm{mg} \cdot \mathrm{mL}^{-1}\right)$ were administered by tail-vein injection $30 \mathrm{~min}$ prior to euthanasia and dye extravasation was used to assess the change in vascular permeability. The fluorescence of the $100 \mu \mathrm{L}$ BALF supernatant (FluobALF) and of $50 \mu \mathrm{L}$ serum (Fluoserum) was measured and permeability volume was expressed in $\mu \mathrm{L}$ :

$$
\begin{gathered}
\text { VPerm }=\left(\text { FluoBALF } \cdot 100 \mu \mathrm{L}^{-1}\right) /\left(\text { Fluoserum } \cdot 50 \mu \mathrm{L}^{-1}\right) \\
\times \text { BALF volume }
\end{gathered}
$$

\section{Protein concentration of the BALF}

The protein content of the BALF supernatants was assessed using the Bio-Rad Protein Assay based on the method of Bradford (Bio-Rad Laboratories $\mathrm{GmbH}$, Munich, Germany). Measurements of absorbance at $595 \mathrm{~nm}$ were performed with a microplate reader (Infinite ${ } 200$ PRO; Tecan Group Ltd, Männedorf, Switzerland).

\section{Histology and electron microscopy}

After completion of the experiment, one part of the right lung was fixed in formalin, embedded in paraffin and stained with Mayer's haematoxylin and eosin for histological examination. Another part of the lung was prepared for scanning electron microscopy as described previously [5].

\section{Neutrophil isolation}

Human neutrophils from healthy donors (males aged 2535 yrs and taking no medication) were isolated as described previously [16]. Neutrophils were incubated with pioglitazone $10 \mu \mathrm{M}$ for 1 or $3 \mathrm{~h}$.

\section{Degranulation}

After incubation with pioglitazone, neutrophils were activated by adding $10 \mathrm{mM} \mathrm{N-formyl-L-methionyl-L-leucyl-L-phenylalanine}$ 

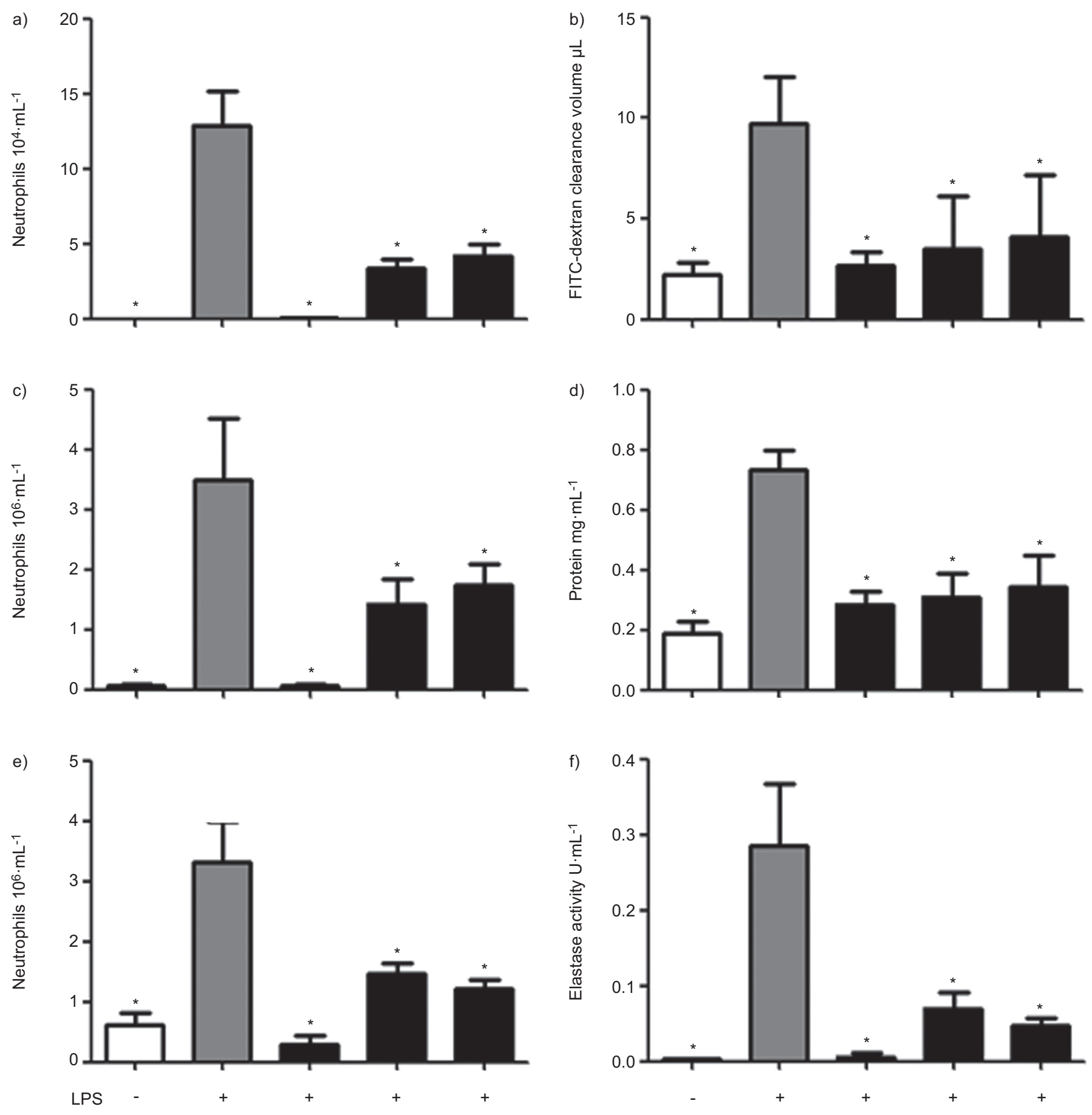

Neutrophil
depletion

Pioglitazone

before ALI

Pioglitazone

after ALI

FIGURE 1. Pioglitazone reduces lipopolysaccharide (LPS)-induced acute lung injury (ALI) by interference with neutrophil recruitment. Mice were challenged with LPS via inhalation and sacrificed $4 \mathrm{~h}$ later. In addition, neutrophils were depleted by antibody injection or mice were treated with piogliatzone ( $2 \mu \mathrm{g}$ per $\mathrm{g}$ body weight) $12 \mathrm{~h}$ and $1 \mathrm{~h}$ before, or $1 \mathrm{~h}$ after LPS exposure as indicated. Quantification of a) alveolar c) interstitial and e) intravascular neutrophils in mice treated as indicated. Lungs were lavaged and b) fluorescein isothiocynate (FITC)-dextran clearance, d) protein concentration and f) elastase activity were assessed in bronchoalveolar lavage fluid of mice treated as indicated. Control $n=8$, LPS $n=10$, LPS+neutrophil depletion $n=9$, and pioglitazone $n=8$. *: $p<0.05$ compared with LPS-treated animals. 

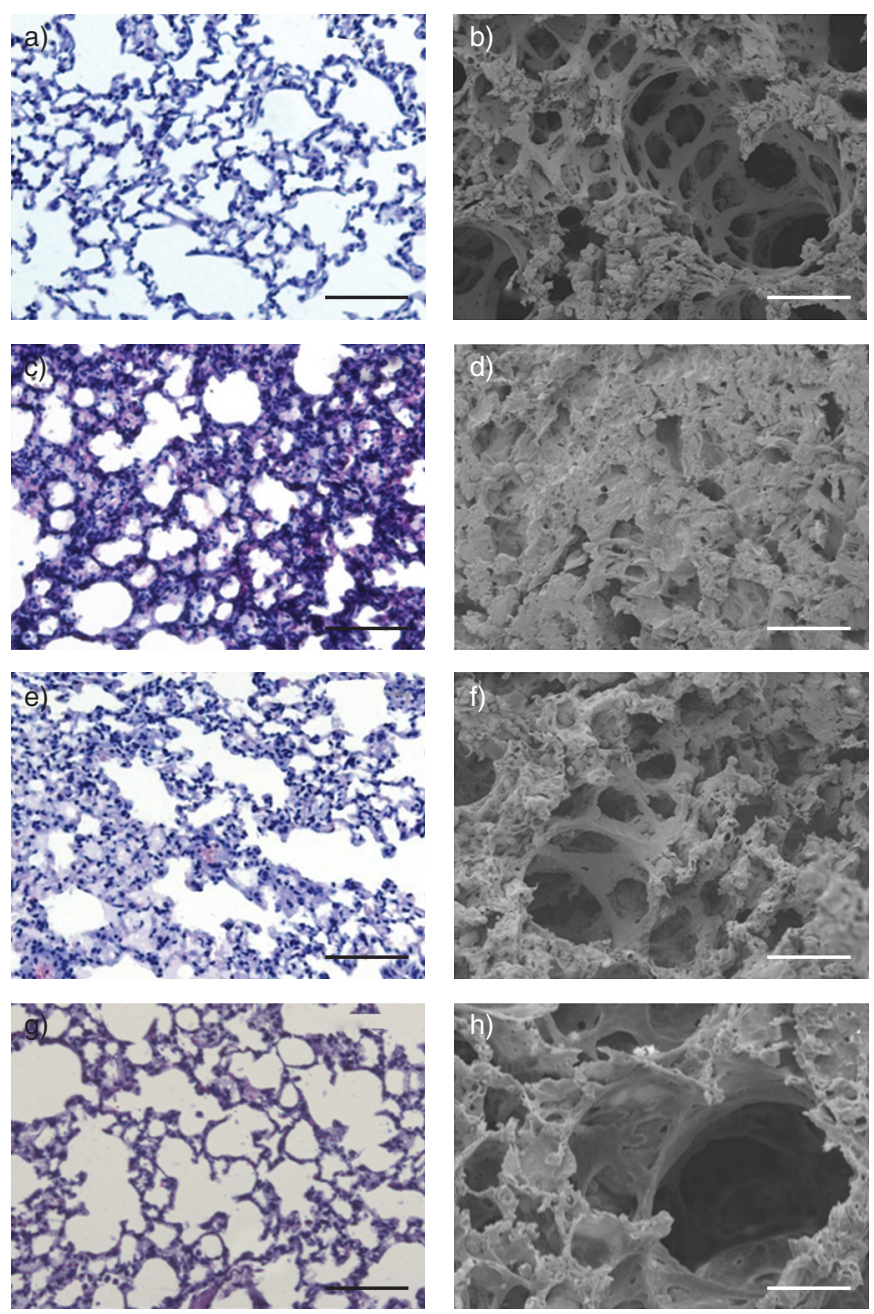

FIGURE 2. Pioglitazone prevents lipopolysaccharide (LPS)-induced structural changes in the lung tissue. a, c, e and g) Representative histological and b, d, f and h) scanning electron microscopic images of lungs from mice treated as follows: a and b) control; c and d) LPS; e and f) pioglitazone before LPS; $g$ and $h$ ) pioglitazone after LPS. $n=5$ for each group. a, c, e and g) Scale bars $=250 \mu \mathrm{m} . \mathrm{b}, \mathrm{d}, \mathrm{f}$ and $\mathrm{h}$ ) Scale bars $=50 \mu \mathrm{m}$

(fMLP; Sigma-Aldrich Co.) and upregulation of CD11b and CD29 was measured after 30 min using BD FACS Canto II.

\section{Flow chamber}

We coated Petri dishes with fibronectin or intercellular adhesion molecule (ICAM)-1 (1 $\mu \mathrm{g} \cdot \mathrm{mL}^{-1}+10 \%$ BSA) for laminar flow chamber. Neutrophils were treated with pioglitazone $(10 \mu \mathrm{M}$ for 1 or $3 \mathrm{~h}$ ). After activation with fMLP, neutrophils were perfused at $1 \mathrm{dyn} \cdot \mathrm{cm}^{-2}$ over fibronectin or ICAM-1 and firmly adherent neutrophils were quantified after $4 \mathrm{~min}$ in multiple fields (a minimum of six fields at $\times 100$ magnification).

\section{Phagocytosis}

Fluorescent Escherichia coli and opsonising reagent (Molecular Probes, Eugene, OR, USA) were reconstituted as indicated by the manufacturer. Immunoglobulin (Ig)G opsonisation was achieved according to the manufacturer's instructions. Complement opsonisation was attained by incubation of bacteria with fresh human serum at $37^{\circ} \mathrm{C}$ for $1 \mathrm{~h}$. Opsonised particles were washed and seeded onto neutrophils, which had been incubated with pioglitazone $10 \mu \mathrm{M}$ for 1 or $3 \mathrm{~h}$. Fluorescence was measured with BD FACS Canto II after $30 \mathrm{~min}$.

\section{Reactive oxygen species}

ROS was detected by dihydrodichlorofluoresceindiacetate (DCF; Molecular Probes) as described previously [17]. Basically, cells were incubated with the profluorescent, lipophilic H2-DCF-DA, which can diffuse through the cell membrane. Reaction with intracellular ROS results in the fluorescent molecule DCF (maximum emission $\sim 530 \mathrm{~nm}$ ), so that DCF fluorescence can be used as a measure for intracellular ROS levels. Fluorescence intensity was quantified with FACS Canto II after $30 \mathrm{~min}$. Similarly, extracellular ROS was measured by singlet oxygen sensor green reagent (Molecular Probes Europe, Leiden, the Netherlands) as recommended by the manufacturer.

\section{Statistics}

All data are expressed as mean \pm SD. Statistical calculations were performed using GraphPad Prism 5 (GraphPad Software Inc., San Diego, CA, USA). Unpaired t-tests, Mann-Whitney test or Kruskal-Wallis test with post hoc Dunn tests were used as appropriate.

\section{RESULTS}

\section{Pioglitazone protects from neutrophil-dependent ALI}

After C57Bl/6 mice were exposed to aerosolised lipopolysaccharide, we observed neutrophil recruitment, plasma leakage, lung (ultra-) structure, and elastase activity in the BALF. Treatment with LPS increased the number of intravascular, interstitial and alveolar neutrophils as analysed by flow cytometry (fig. S2) of lung homogenates and BALF (fig. 1). Furthermore, the protein concentration, as well as the clearance of fluorescent dextran, increased in the BALF with the LPS treatment, thereby indicating enhanced plasma leakage and oedema formation. Moreover, the activity of neutrophil-derived elastase, a protease important in ALI, was elevated in LPS-treated animals (fig. 1). Neutrophil depletion abolishes alveolar fluid efflux and structural changes confirming the previously described importance of neutrophils in ALI (fig. 1). To test the potential role of pioglitazone in this model of neutrophil-mediated ALI, mice were treated with pioglitazone prior to LPS exposure. In these experiments we found that pioglitazone reduced the recruitment of neutrophils after LPS inhalation in the intravascular, interstitial and alveolar compartment of the lung (fig. 1) and prevented enhanced pulmonary vascular leakage indicated by reduced protein content of the BALF and FITC-dextran clearance volume (fig. 1). In addition, treatment with pioglitazone $1 \mathrm{~h}$ after induction of ALI exhibited similar effects (fig. 1). Histological and ultrastructural analyses of lung following LPS exposure revealed alveolar septal thickening, accumulation of inflammatory cells in the interstitium and the alveoli, and influx of protein-rich fluid into the alveolar space as compared to control mice exposed to aerosolised saline solution. Pioglitazone both before (fig. 2) and after (data not shown) LPS inhalation abrogated histological alterations of this kind, further supporting its protective role in neutrophil-mediated ALI. 

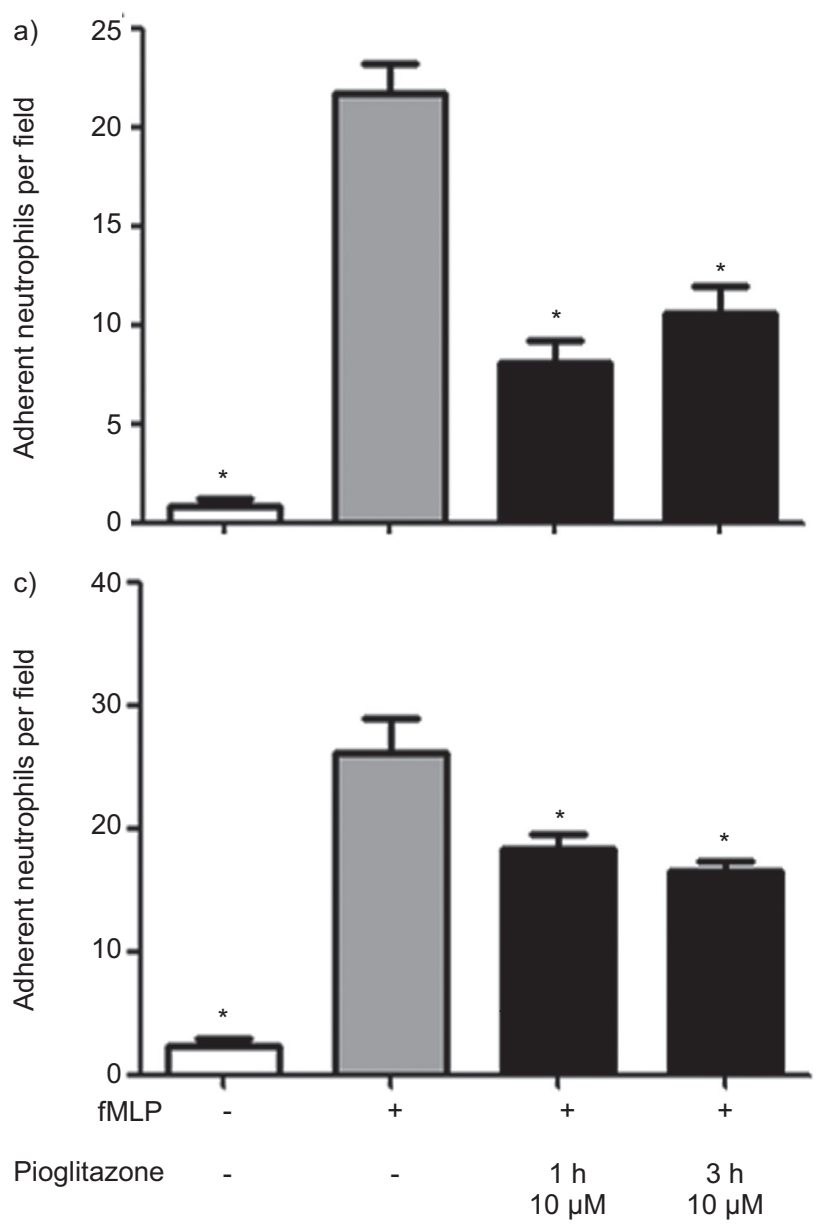
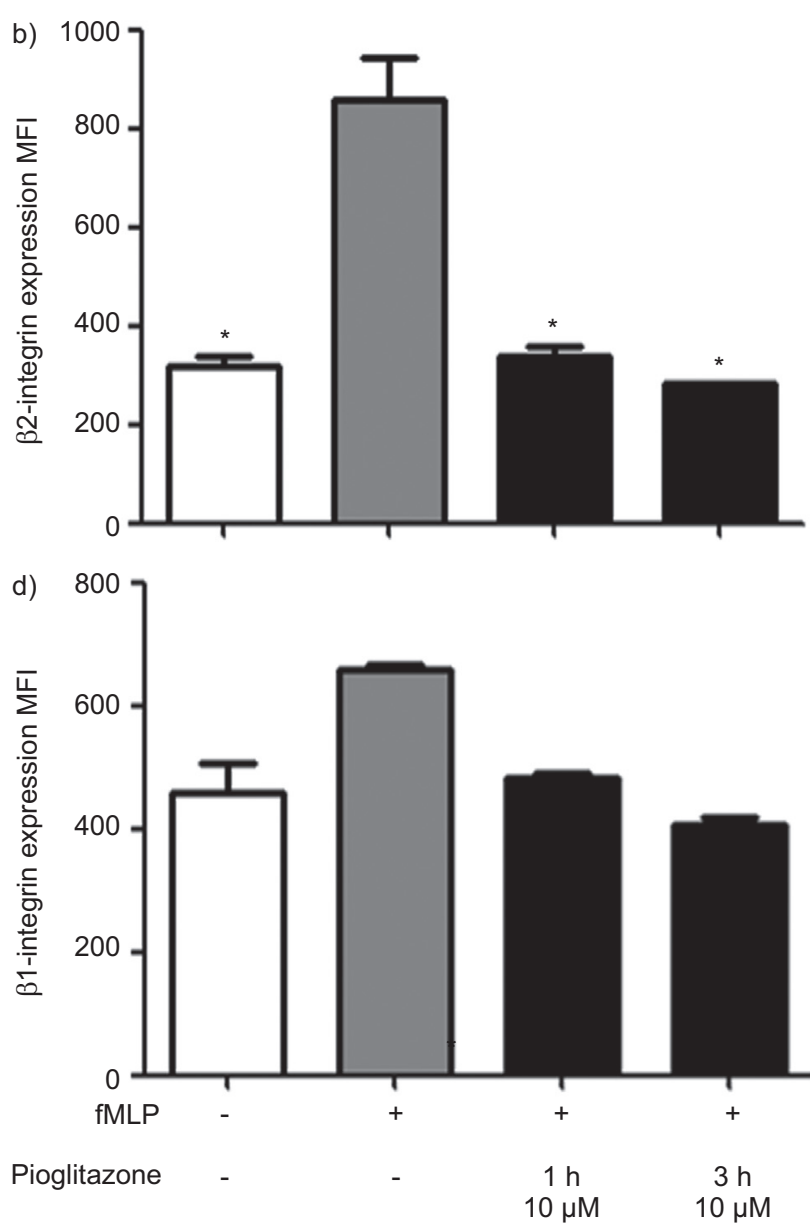

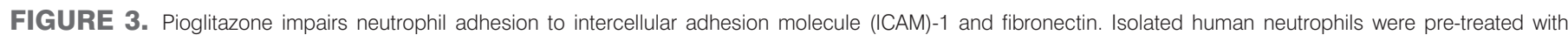

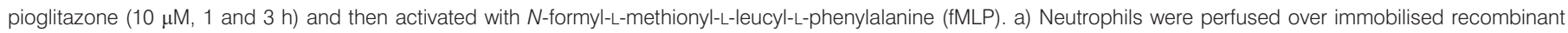

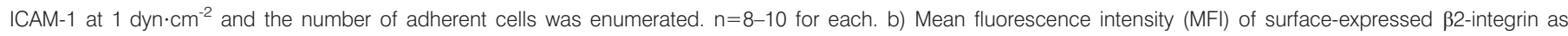

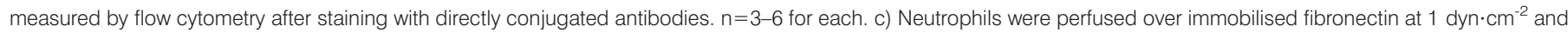

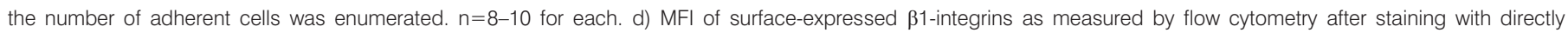
conjugated antibodies. $n=3-6$ for each. *: $p<0.05$ compared to the fMLP group.

\section{Pioglitazone reduces neutrophil adhesion to ICAM-1 and fibronectin}

Our in vivo data highlight the direct reduction of neutrophil recruitment by treatment with pioglitazone. To further confirm this notion, we analysed the effect of pioglitazone on adhesion of isolated human neutrophils perfused over immobilised ICAM-1 (fig. 3). Treatment of neutrophils with pioglitazone for $1 \mathrm{~h}$ and $3 \mathrm{~h}$ at $10 \mu \mathrm{M}$ severely diminished adhesion to ICAM-1. For neutrophils to firmly adhere to ICAM-1, the upregulation of $\beta 2$-integrins from secretory vesicles is a prerequisite. Such mobilisation is mediated by secretagogues, such as the bacterial wall peptide fMLP. Consequently, we analysed the effect of pioglitazone on fMLP-induced $\beta 2$-integrin upregulation on neutrophils. After activation of neutrophils with fMLP, expression of $\beta 2$-integrin was significantly elevated (fig. 3). Pioglitazone $(10 \mu \mathrm{M}$ for 1 or $3 \mathrm{~h})$ significantly reduced the fMLP-induced expression of $\beta 2$-integrins (fig. 3), thus offering an explanation for decreased adhesion to ICAM-1 following pioglitazone treatment.
As $\beta 1$-integrins are crucial for extravascular locomotion of neutrophils, we tested the effect of pioglitazone on $\beta 1$-integrin upregulation and neutrophil adhesion to the $\beta_{1}$-integrin substrate fibronectin. Flow chamber experiments revealed significantly reduced adhesion of neutrophils to fibronectin after pretreatment with pioglitazone $(10 \mu \mathrm{M})$ for either 1 or $3 \mathrm{~h}$ (fig. 3). Treatment of neutrophils with fMLP resulted in a trend to increased surface expression of the fibronectin ligand $\alpha 5 \beta 1$-integrin, an effect fully reversed by pretreatment with pioglitazone (fig. 3).

\section{Pioglitazone does not impair neutrophil antimicrobial activity}

Besides their contribution to ALI, neutrophils display important antibacterial effector functions in bacterial infections. To analyse if the beneficial anti-inflammatory effect of pioglitazone does not negatively affect these functions, we tested the capacity of pioglitazone-treated neutrophils to phagocytose bacteria. Phagocytosis of IgG-opsonised (fig. 4a) or complementopsonised (fig. 4b) FITC-labelled E. coli was assessed by flow 

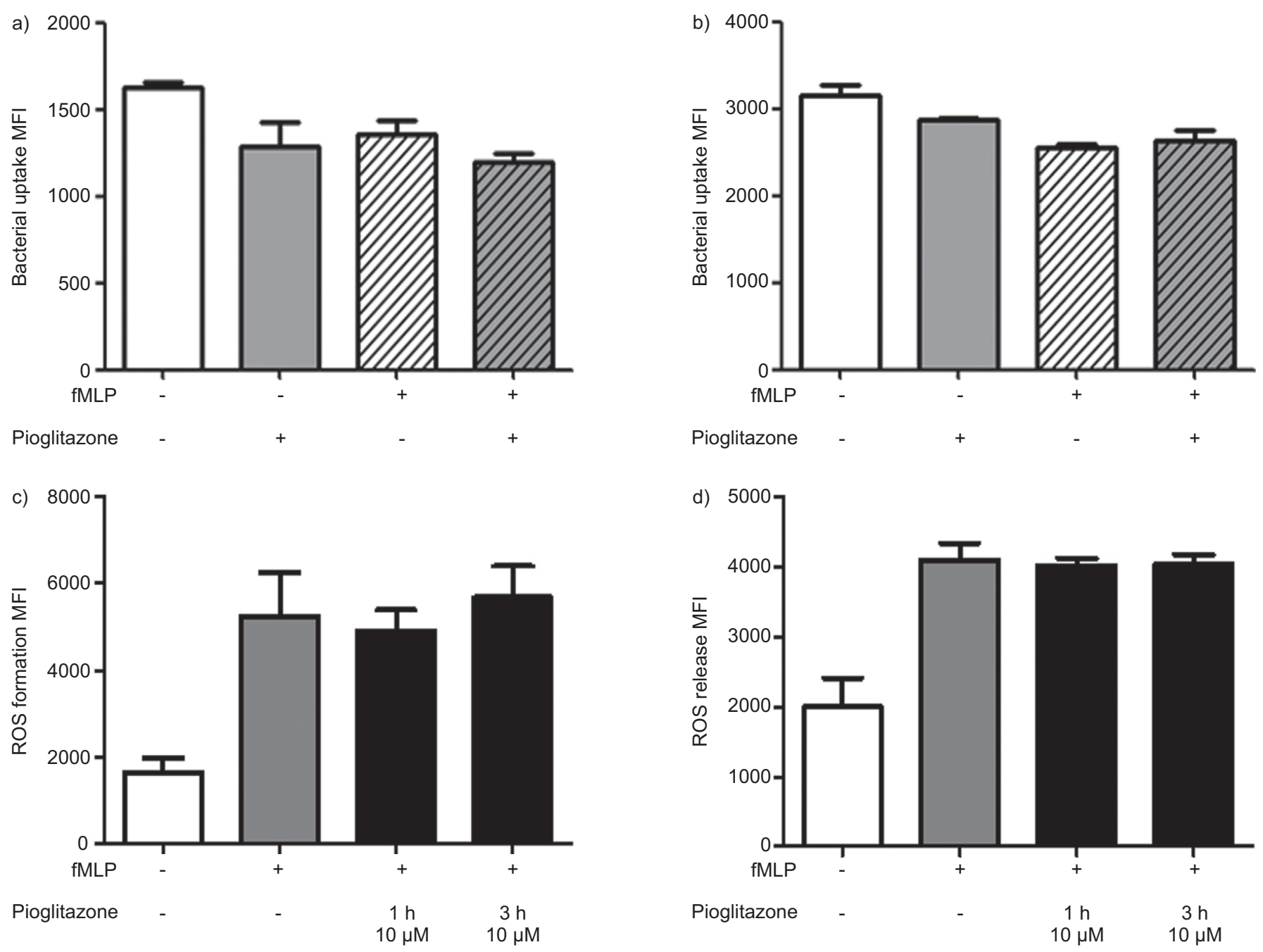

FIGURE 4. Pioglitazone does not affect neutrophil antimicrobial activity. Bacterial uptake of fluorescent a) immunoglobulin G- or b) complement-opsonised Escherichia coli by activated or resting neutrophils as assessed by flow cytometry. Neutrophils were activated with $N$-formyl-L-methionyl-L-leucyl-L-phenylalanine (fMLP) and pre-treated with pioglitazone as indicated. $\mathrm{n}=4$. Isolated human neutrophils were pre-treated with pioglitazone $(10 \mu \mathrm{M}, 1$ and $3 \mathrm{~h})$. c) Neutrophils were labelled with the sensitive dye $2^{\prime}, 7^{\prime}$-dichlorodihydrofluorescein diacetate and reactive oxygen species (ROS) formation was recorded by flow cytometry following fMLP stimulation. Data indicate mean fluorescence intensity (MFI) 30 min after fMLP exposure. $n=6$ for each. d) Neutrophils were labelled with singlet oxygen green as a marker of extracellular ROS release. Data indicate MFI 30 min after fMLP exposure. $\mathrm{n}=6$ for each. Data are presented as mean $\pm \mathrm{SD}$.

cytometry. Whereas the bacterial uptake of neutrophils increased after complement opsonisation in comparison to the IgG opsonisation, pioglitazone did not significantly alter bacterial uptake.

Further to adhesion and migration, neutrophils contribute to ALI by release of ROS. However, ROS also displays important antimicrobial functions in neutrophils. Hence, we investigated the effect of pioglitazone on ROS formation and release of isolated human neutrophils induced by fMLP. After isolation of neutrophils from healthy donors, neutrophils were incubated with pioglitazone $(10 \mu \mathrm{M})$ for 1 or $3 \mathrm{~h}$. fMLP clearly induced formation and release of ROS over time. However, pioglitazone pre-treatment failed to affect ROS formation (fig. 4c) and release (fig. 4d), thus implying that pioglitazone does not impair neutrophil antimicrobial activity and that the protective effect of pioglitazone does not stem from effects on ROS release.

\section{DISCUSSION}

Despite all innovations in intensive care medicine, ALI induced by Gram-negative bacteria remains a major challenge. In our study, we demonstrate a beneficial effect of pioglitazone in ALI treatment as indicated by reduced oedema formation and neutrophil infiltration, both of which are key events during development of ALI.

PPAR- $\alpha$ and $-\gamma$ agonists have been developed for treatment of dyslipidaemia and type 2 diabetes. However, recent studies have revealed additional beneficial effects in atherosclerosis and inflammatory diseases, which are partly explained by stabilisation of endothelial function $[18,19]$. The protective effect of PPAR- $\alpha$ agonists of the fibrate class in LPS-induced lung injury has previously been established [20]. With the documented importance of PPAR- $\gamma$ in control of neutrophil migration [21], we investigated the effect of glitazones, which might directly reduce the activation and recruitment of the 
neutrophils, a process that importantly contributes to tissue damage in ALI [3]. Consequently, we analysed the effects of pioglitazone on neutrophil activity. The importance of neutrophil infiltration in LPS-induced ALI is substantiated in models where neutrophil adhesion or migration is impaired. In this context, it was shown that lack of CXCR2 or a blockade of $\beta_{2}$-integrins protects from ALI [22, 23]. In our study, pioglitazone prevented intravascular neutrophil adhesion and lung infiltration. As this was addressed in an in vitro assay in the absence of other cell types but in the presence of substrates typically involved in neutrophil adhesion and migration, we conclude that the in vivo effects may, in a large part, relate to direct interference with surface expression of $\beta 1$-integrins and $\beta 2$-integrins. Our results are consistent with a previous study that revealed reduced monocyte adhesion on endothelial cells indicating a protective role in acute inflammation of pioglitazone [24]. Interestingly, in our study, we found similar effect of pioglitazone treatment after LPS inhalation in comparison to the treatment before LPS inhalation. This is intriguing as this mode of treatment probably relies on rapidly occurring anti-inflammatory activities. A possible explanation might be the reduced expression of endothelial cell adhesion molecules [25, 26]. Especially decreased expression of P-selectin following treatment with glitazones may offer an explanation for reduced neutrophil recruitment [26]. Furthermore, reduced oxidative stress [27] and decreases in the release of lipid mediators [28] in response to glitazones may offer alternative explanations for reduced neutrophil lung infiltration when treatment is initiated after LPS inhalation.

Rapid upregulation of $\beta 2$-integrins on neutrophils is typically a result of mobilisation of preformed granules. $\beta 2$-integrins are localised in secretory vesicles, a compartment discharged when neutrophil-endothelial interaction is established. Secretory vesicles are also rich in azurocidin [29], a protein previously associated with neutrophil-mediated permeability changes $[5,30,31]$. Hence, reduced surface-expression of $\beta 2-$ integrins following fMLP stimulation not only explains reduced adhesive capacity, but may also point to impaired release of granule proteins relevant to ALI. Consistent with this, we found lower elastase activity in BALF from mice treated with pioglitazone. Elastase aggravates ALI by increasing endothelial and epithelial permeability $[32,33]$, proteolytic cleavage of surfactant proteins [34] and induction of apoptosis [35]. The in vivo importance of neutrophil elastase in ALI is further corroborated in studies using elastase-deficient mice [36] or employing specific inhibitors [37]. Although the release of ROS is an important antimicrobial mechanism, overproduction of ROS can cause tissue damage in sepsis and ALI [38]. In animal models of ALI, neutrophil-derived ROS cause lung injury, as shown by histological examination and permeability measurements $[39,40]$. In addition, it has been shown that ROS can disrupt intercellular tight junctions of the endothelium by phosphorylation of focal adhesion kinase [41]. Hence, deficiency or blockade of reduced nicotinamide adenine dinucleotide phosphate oxidase prevents ALI [38, 42, 43]. However, in our study, pioglitazone failed to affect ROS release. Hence, the protective effect of pioglitazone appears to primarily arise from decreases in neutrophil degranulation, adhesion and recruitment.
After migration, neutrophils are irreplaceable in bacterial clearance, much of which is mediated by phagocytosis and intracellular bacterial killing [44]. Data from our study indicate that pioglitazone does not negatively affect bacterial uptake and clearance, as assessed by ROS formation experiments. Hence, these data suggest that pioglitazone might not impair clearance during bacterial infections and, thus, further support its clinical applicability. However, further in vivo studies are required to evaluate the effect of pioglitazone on bacterial clearance in a broader setting.

\section{Conclusion}

Pioglitazone attenuates recruitment and activation of neutrophils in a model of ALI and, thereby, displays beneficial effects. Moreover, pioglitazone treatment after onset of ALI was as effective as treatment before onset of ALI, implicating a potential role for glitazones in the management of ALI.

\section{SUPPORT STATEMENT}

This study was supported by the Deutsche Forschungsgemeinschaft, the German Heart Foundation, the Else-Kröner-Fresenius Foundation and the B. Braun Foundation.

\section{STATEMENT OF INTEREST}

None declared.

\section{ACKNOWLEDGEMENTS}

The authors wish to acknowledge X. Balaj, S. Roubrocks and S. Winkler (all, Institute for Molecular Cardiovascular Research (IMCAR), RWTH Aachen, Aachen, Germany) for their excellent technical assistance.

\section{REFERENCES}

1 Ware LB, Matthay MA. The acute respiratory distress syndrome. N Engl J Med 2000; 342: 1334-1349.

2 Matute-Bello G, Frevert CW, Martin TR. Animal models of acute lung injury. Am J Physiol Lung Cell Mol Physiol 2008; 295: L379-L399.

3 Grommes J, Soehnlein O. Contribution of neutrophils to acute lung injury. Mol Med 2011; 17: 293-307.

4 Looney MR, Su X, Van Ziffle JA, et al. Neutrophils and their Fcy receptors are essential in a mouse model of transfusion-related acute lung injury. J Clin Invest 2006; 116: 1615-1623.

5 Soehnlein O, Oehmcke S, Ma X, et al. Neutrophil degranulation mediates severe lung damage triggered by streptococcal M1 protein. Eur Respir J 2008; 32: 405-412.

6 Gautam N, Olofsson AM, Herwald H, et al. Heparin-binding protein (HBP/CAP37): a missing link in neutrophil-evoked alteration of vascular permeability. Nat Med 2001; 7: 1123-1127.

7 Bdeir K, Higazi A, Kulikovskaya I, et al. Neutrophil alphadefensins cause lung injury by disrupting the capillary-epithelial barrier. Am J Respir Crit Care Med 2010; 181: 935-946.

8 Pham CT. Neutrophil serine proteases: specific regulators of inflammation. Nat Rev Immunol 2006; 6: 541-550.

9 Massberg S, Grahl L, von Bruehl ML, et al. Reciprocal coupling of coagulation and innate immunity via neutrophil serine proteases. Nat Med 2010; 16: 887-896.

10 Brown JD, Plutzky J. Peroxisome proliferator-activated receptors as transcriptional nodal points and therapeutic targets. Circulation 2007; 115: 518-533.

11 Ricote M, Li AC, Willson TM, et al. The peroxisome proliferatoractivated receptor- $\gamma$ is a negative regulator of macrophage activation. Nature 1998; 391: 79-82.

12 Jiang C, Ting AT, Seed B. PPAR- $\gamma$ agonists inhibit production of monocyte inflammatory cytokines. Nature 1998; 391: 82-86. 
13 Becker J, Delayre-Orthez C, Frossard N, et al. Regulation of inflammation by PPARs: a future approach to treat lung inflammatory diseases? Fundam Clin Pharmacol 2006; 20: 429-447.

14 Park SJ, Lee KS, Kim SR, et al. Peroxisome proliferator-activated receptor $\gamma$ agonist down-regulates IL-17 expression in a murine model of allergic airway inflammation. J Immunol 2009; 183: 3259-3267.

15 Aoki Y, Maeno T, Aoyagi K, et al. Pioglitazone, a peroxisome proliferator-activated receptor $\gamma$ ligand, suppresses bleomycininduced acute lung injury and fibrosis. Respiration 2009; 77: 311-319.

16 Soehnlein O, Kai-Larsen Y, Frithiof R, et al. Neutrophil primary granule proteins HBP and HNP1-3 boost bacterial phagocytosis by human and murine macrophages. J Clin Invest 2008; 118: 3491-3502.

17 Soehnlein O, Kenne E, Rotzius $\mathrm{P}$, et al. Neutrophil secretion products regulate anti-bacterial activity in monocytes and macrophages. Clin Exp Immunol 2008; 151: 139-145.

18 Campia U, Matuskey LA, Panza JA. Peroxisome proliferatoractivated receptor- $\gamma$ activation with pioglitazone improves endothelium-dependent dilation in nondiabetic patients with major cardiovascular risk factors. Circulation 2006; 113: 867-875.

19 Marx N, Wöhrle J, Nusser T, et al. Pioglitazone reduces neointima volume after coronary stent implantation: a randomized, placebocontrolled, double-blind trial in nondiabetic patients. Circulation 2005; 112: 2792-2798.

20 Schaefer MB, Pose A, Ott J, et al. Peroxisome proliferatoractivated receptor-alpha reduces inflammation and vascular leakage in a murine model of acute lung injury. Eur Respir J 2008; 32: 1344-1353.

21 Reddy RC, Narala VR, Keshamouni VG, et al. Sepsis-induced inhibition of neutrophil chemotaxis is mediated by activation of peroxisome proliferator-activated receptor- $\gamma$. Blood 2008; 112: $4250-4258$.

22 Belperio JA, Keane MP, Burdick MD, et al. Critical role for CXCR2 and CXCR2 ligands during the pathogenesis of ventilator-induced lung injury. J Clin Invest 2002; 110: 1703-1716.

23 Moreland JG, Fuhrman RM, Pruessner JA, et al. CD11b and intercellular adhesion molecule-1 are involved in pulmonary neutrophil recruitment in lipopolysaccharide-induced airway disease. Am J Respir Cell Mol Biol 2002; 27: 474-480.

24 Haraguchi G, Kosuge H, Maejima Y, et al. Pioglitazone reduces systematic inflammation and improves mortality in apolipoprotein E knockout mice with sepsis. Intensive Care Med 2008; 34: 1304-1312.

25 Imamoto E, Yoshida N, Uchiyama K, et al. Inhibitory effect of pioglitazone on expression of adhesion molecules on neutrophils and endothelial cells. Biofactors 2004; 20: 37-47.

26 Cuzzocrea S, Pisano B, Dugo L, et al. Rosiglitazone, a ligand of the peroxisome proliferator-activated receptor- $\gamma$, reduces acute inflammation. Eur J Pharmacol 2004; 483: 79-93.

27 Kaundal RK, Iyer S, Kumar A, et al. Protective effects of pioglitazone against global cerebral ischemic-reperfusion injury in gerbils. J Pharmacol Sci 2009; 109: 361-367.
28 Yamashita M, Kushihara M, Hirasawa N, et al. Inhibition by troglitazone of the antigen-induced production of leukotrienes in immunoglobulin E-sensitized RBL-2H3 cells. Br J Pharmacol 2000; 129: 367-373.

29 Soehnlein O, Lindbom L. Neutrophil-derived azurocidin alarms the immune system. J Leukoc Biol 2009; 85: 344-351.

30 Gautam N, Herwald $\mathrm{H}$, Hedqvist $\mathrm{P}$, et al. Signaling via $\beta 2$ integrins triggers neutrophil-dependent alteration in endothelial barrier function. J Exp Med 2000; 191: 1829-1839.

31 Herwald H, Cramer H, Mörgelin M, et al. M protein, a classical bacterial virulence determinant, forms complexes with fibrinogen that induce vascular leakage. Cell 2004; 116: 367-379.

32 DiStasi MR, Ley K. Opening the flood-gates: how neutrophilendothelial interactions regulate permeability. Trends Immunol 2009; 30: 547-556.

33 Ginzberg HH, Cherapanov V, Dong Q, et al. Neutrophil-mediated epithelial injury during transmigration: role of elastase. Am J Physiol Gastrointest Liver Physiol 2001; 281: G705-G717.

34 Hirche TO, Crouch EC, Espinola M, et al. Neutrophil serine proteinases inactivate surfactant protein $\mathrm{D}$ by cleaving within a conserved subregion of the carbohydrate recognition domain. J Biol Chem 2004; 279: 27688-27698.

35 Suzuki T, Yamashita C, Zemans RL, et al. Leukocyte elastase induces lung epithelial apoptosis via a PAR-1-, NF-kB-, and p53dependent pathway. Am J Respir Cell Mol Biol 2009; 41: 742-755.

36 Tkalcevic J, Novelli M, Phylactides M, et al. Impaired immunity and enhanced resistance to endotoxin in the absence of neutrophil elastase and cathepsin G. Immunity 2000; 12: 201-210.

37 Kawabata K, Hagio T, Matsumoto S, et al. Delayed neutrophil elastase inhibition prevents subsequent progression of acute lung injury induced by endotoxin inhalation in hamsters. Am J Respir Crit Care Med 2000; 161: 2013-2018.

38 Carnesecchi S, Deffert C, Pagano A, et al. NADPH oxidase-1 plays a crucial role in hyperoxia-induced acute lung injury in mice. Am J Respir Crit Care Med 2009; 180: 972-981.

39 Auten RL, Richardson RM, White JR, et al. Nonpeptide CXCR2 antagonist prevents neutrophil accumulation in hyperoxiaexposed newborn rats. J Pharmacol Exp Ther 2001; 299: 90-95.

40 Auten RL, Whorton MH, Mason SN. Blocking neutrophil influx reduces DNA damage in hyperoxia-exposed newborn rat lung. Am J Respir Cell Mol Biol 2002; 26: 391-397.

41 Chiarugi P, Pani G, Giannoni E, et al. Reactive oxygen species as essential mediators of cell adhesion: the oxidative inhibition of a FAK tyrosine phosphatase is required for cell adhesion. J Cell Biol 2003; 161: 933-944.

42 Kubo H, Morgenstern D, Quinian WM, et al. Preservation of complement-induced lung injury in mice with deficiency of NADPH oxidase. J Clin Invest 1996; 97: 2680-2684.

43 Wang W, Suzuki Y, Tanigaki T, et al. Effect of the NADPH oxidase inhibitor apocynin on septic lung injury in guinea pigs. Am J Respir Crit Care Med 1994; 150: 1449-1452.

44 Soehnlein O. Direct and alternative antimicrobial mechanisms of neutrophil-derived granule proteins. J Mol Med 2009; 87: 1157-1164. 